Revista Arbitrada Interdisciplinaria de Ciencias de la Salud. SALUD Y VIDA

Volumen 4. Número 7. Año 4. Enero - Junio 2020

Hecho el depósito de Ley: FA2016000010

ISSN: $2610-8038$

FUNDACIÓN KOINONIA (F.K)

Santa Ana de Coro, Venezuela.

Sandra c. Romero de Jaimes

http://dx.doi.org/10.35381/s.v.v4i7.664

\title{
Los programas nacionales de formación una política educativa para la transformación social de la salud
}

\section{National training programs an educational policy for social transformation of health}

\author{
Sandra C. Romero de Jaimes \\ sandrar.unefm@gmail.com \\ Universidad Nacional Experimental Francisco de Miranda, Santa Ana de Coro \\ Venezuela \\ https://orcid.org/0000-0003-1965-0102
}

Recibido: 15 de noviembre de 2019

Aprobado: 20 de diciembre de 2019

\section{RESUMEN}

La educación como derecho fundamental de todo ser humano permite el desarrollo como ciudadano para lograr una calidad de vida. Esta debe responder a las exigencias del mundo de hoy, en donde las instituciones universitarias asumen una misión que les lleva a revaluar sus funciones y su pertinencia social con el momento histórico. El objetivo de esta investigación fue analizar a través de una metodología documental los Programas Nacionales de Formación como política educativa para la transformación social de la salud. Se concluyó que las universidades a través de los Programas Nacionales de Formación del área de la salud responden a las necesidades que se plantean en el Sistema Nacional de Salud formando profesionales con calidad educativa y con responsabilidad social, para una sociedad más humanizada, en el desarrollo individual y colectivo. Alcanzar el progreso de forma sostenible y sustentable atendiendo los derechos fundamentales de la salud para la prevención de enfermedades.

Descriptores: Programas Nacionales de Formación; calidad educativa; responsabilidad social; transformación de la salud.

\begin{abstract}
Education as a fundamental right of every human being allows development as a citizen to achieve a quality of life. This should respond to the demands of today's world, where university institutions assume a mission that leads them to revalue their functions and their social relevance to the historical moment. The objective of this research was to analyze
\end{abstract}




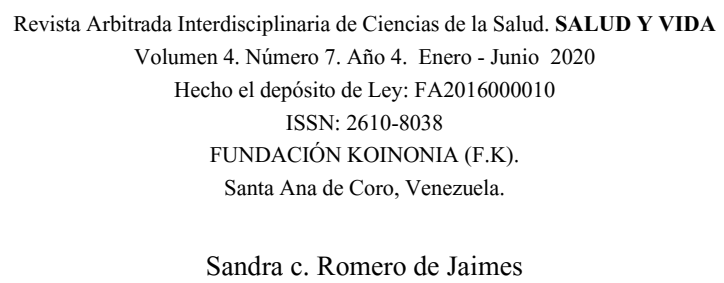

the National Training Programs as an educational policy for the social transformation of health through a documentary methodology. It was concluded that universities through the National Training Programs of the health area respond to the needs that arise in the National Health System by training professionals with educational quality and social responsibility, for a more humanized society, in development individual and collective Achieve progress in a sustainable and sustainable way attending to the fundamental rights of health for disease prevention.

Descriptors: National training programs; educational quality; social responsibility; health transformation.

\section{INTRODUCCIÓN}

La educación se constituye en un derecho que busca la formación del individuo para la vida, por lo tanto, se asienta dentro de los derechos políticos, económicos, sociales y culturales de una sociedad. Es por ello que una educación de calidad y pertinencia tiene y debe ser la condición esencial para superar la pobreza y las diferentes problemáticas que se dan a escala mundial, para de esta manera poder avanzar hacia una mayor igualdad de oportunidades entre los ciudadanos. En consecuencia, el derecho a la educación implica para los Estados la responsabilidad de asegurar acceso, progresión y culminación de los distintos niveles educativos en todas las etapas.

Razón por lo cual, a nivel universitario se debe hacer énfasis de forma constante y permanente en la búsqueda de una educación de calidad y con pertinencia por lo que se realizan reformas en la educación superior tanto en el plano nacional como en el internacional para lograr un prestigio académico ante las exigencias de los gobiernos, las comunidades científicas, los colegios profesionales y por supuesto, la sociedad.

\section{PLANTEAMIENTO DEL PROBLEMA}

La educación ha presentado diversas connotaciones que han estado en relación directa con el momento histórico que se vive, además de presentar particularidades que tienen relación directa con la cultura teniendo en cuenta aspectos tan importantes que están 


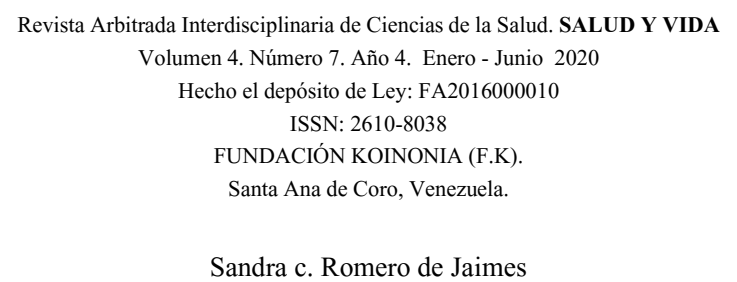

vinculados con lo histórico, los valores, la ética y con el profesional que se requiere para el desarrollo sostenible y sustentable en cada nación.

En esta perspectiva, a escala mundial se demanda la formación de profesionales con pertinencia social y calidad académica que conlleve a lograr el desarrollo sostenible y sustentable del mundo de hoy. Por lo tanto, la educación superior asume un papel importante porque debe ser el promotor del cambio social que conlleve a la generación de conocimientos que permitan el abordaje de las problemáticas a nivel mundial, específicamente en aspectos como la seguridad alimentaria, el cambio climático, la gestión del agua, el diálogo intercultural, las energías renovables y la salud pública. (UNESCO, 2009).

Por tal motivo, se deben evaluar de forma continua los programas que se implementan para poder determinar su pertinencia, coherencia y calidad para dar respuesta efectiva a las demandas de la sociedad y egresar profesionales que contribuyan al desarrollo sostenible y sustentable de las naciones. Conviene considerar, que la calidad educativa es multidimensional y requiere la participación activa y comprometida de docentes, padres o representantes, autoridades, especialistas, la sociedad y del Estado como regente para fomentar la paz, la libertad y el desarrollo de las naciones a través de la práctica contextualizada que permitirá modificar la realidad y propiciará nuevas experiencias lo cual supone un avance cualitativo en el proceso de la responsabilidad social como modo de gestión integral en la educación que permita responder a las necesidades de transformación de la sociedad, (Alvarado, 2011)

Es importante subrayar, que la educación como derecho fundamental tiene una trascendental función en el ámbito social, porque es la que moviliza todo lo necesario para que el sujeto entre al mundo y se sostenga en él. En esta perspectiva, la Conferencia Regional de América Latina y El Caribe (2008), declara que:

Las instituciones de Educación Superior deben avanzar en la configuración de una relación más activa con sus contextos. La calidad está vinculada a la pertinencia y la responsabilidad con el desarrollo sostenible de la sociedad. Ello exige impulsar un modelo académico caracterizado por la indagación de los problemas en sus contextos; la producción y transferencia del valor social 


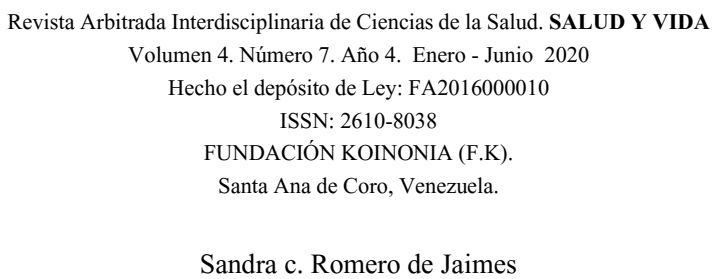

de los conocimientos; el trabajo conjunto con las comunidades; una investigación científica, tecnológica, humanística y artística fundada en la definición explícita de problemas a atender, de solución fundamental para el desarrollo del país o la región, y el bienestar de la población (p.15).

Por consiguiente, se ratifica la necesidad que se tiene de que las universidades, institutos y colegios universitarios trabajen de cara al contexto y al momento histórico con sentido de pertinencia, con apertura a nuevas formas de aprendizaje que se correspondan con las características de vida del trabajador y con modalidades curriculares concretamente flexibles que ofrezcan mejorar y actualizar la formación profesional, además de la aceptación de que lo inter y lo transdisciplinario es esencial para comprender la compleja realidad que se vive.

De tal manera, que se requiere que las universidades inicien un proceso de cambio institucional para estar en sintonía con las transformaciones que se han originado en esta nueva época. Debe señalarse que, estas exigencias de transformación se proyectan en la Educación Universitaria de la República Bolivariana de Venezuela con el fin de hacer acertada su adaptación a los cambios que ha experimentado la sociedad en las últimas décadas, por lo que se han implementación un conjunto de políticas educativas cuyos propósitos son dar respuesta efectiva a la nación con la formación de profesionales con calidad educativa y responsabilidad social.

Es por ello, que dentro de estas políticas esta la creación de los Programas Nacionales de Formación del área de la salud. Sin embargo, es necesario tener muy en cuenta la calidad educativa y la responsabilidad social por lo que se deben realizar revisiones periódicas que permitan discutir y analizar el accionar pedagógico para la construcción de una sociedad más justa, equitativa y que permita el desarrollo individual y colectivo para el logro de la paz, la libertad y la sustentabilidad.

Ante esta realidad existen las siguientes interrogantes; ¿Se realizan procesos de evaluación de los Programas Nacionales de Formación del área de la salud en la UNEFM en cuanto a la calidad educativa? ¿Existe pertinencia social en los Programas Nacionales de Formación del área de la salud de la UNEFM? 
Revista Arbitrada Interdisciplinaria de Ciencias de la Salud. SALUD Y VIDA

Volumen 4. Número 7. Año 4. Enero - Junio 2020

Hecho el depósito de Ley: FA2016000010

ISSN: 2610-8038

FUNDACIÓN KOINONIA (F.K)

Santa Ana de Coro, Venezuela.

Sandra c. Romero de Jaimes

\section{Objetivos de la investigación Objetivo General}

Analizar la calidad educativa y la pertinencia social de los Programas Nacionales de Formación del área de la salud de la UNEFM para el logro de la transformación universitaria.

\section{Objetivos Específicos}

Efectuar una revisión documental sobre el modelo curricular de los Programas Nacionales de Formación del área de la salud en la UNEFM.

Identificar los procesos de evaluación de los Programas Nacionales de Formación del área de la salud de la UNEFM en cuanto a su pertinencia social.

Caracterizar los fundamentos y principios que rigen los Programas Nacionales de Formación del área de la salud en cuanto a su pertinencia social.

\section{MARCO TEÓRICO}

\section{Educación universitaria con calidad}

La calidad de la educación superior debe estar dada por su accionar social lo cual conlleva a procesos de trasformación en todos los ámbitos del individuo que se forma como profesional. En este sentido, se tiene que la calidad de la educación engloba conceptos como relevancia, pertinencia y eficiencia. Donde la relevancia esta en relación directa con las necesidades sociales y sus cambios, y se procura establecer un tipo de educación adecuada que permita satisfacerla.

Es por ello, que Ramsden (2007) considera que una enseñanza de calidad en educación superior debe cambiar la manera como los estudiantes comprenden, experimentan o conceptualizan el mundo que les rodea y propone seis principios de una enseñanza efectiva en educación superior, que son:

1) Despertar el interés y los deseos de aprender por parte de los estudiantes, donde ellos acepten el esfuerzo que va requerir.

2) Preocupación y respeto por el aprendizaje del alumno, es decir, todas sus acciones deben estar encaminadas a lograr cambios en su comprensión del mundo. 


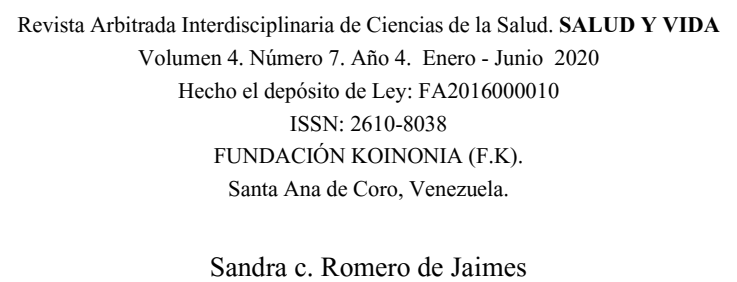

3) ofrecer una realimentación adecuada y una justa evaluación, sobre todo la primera, ya que es la característica de una enseñanza eficiente más citada por los estudiantes.

4) metas claras y retos intelectuales: al estudiante le debe quedar claro lo que se espera de él y alcanzar esa finalidad debe implicar un desafío.

5) fomento de la independencia y control de su aprendizaje por parte del alumno y su activo involucramiento: la finalidad para el estudiante es que al término del proceso instruccional sea un aprendiz autónomo y autorregulado.

6) aprender de los estudiantes, lo que implica que el maestro debe ser humilde y estar dispuestos a conocer cosas nuevas; ser generoso para compartir lo que sabe y conocer a sus alumnos para adaptar sus conocimientos a sus características, expectativas y deseos, y no al revés.

De tal manera, que la calidad educativa de los Programas Nacionales de Formación del área de la salud debe estar orientada hacia la innovación, que este en correspondencia con los objetivos de la universidad, que tenga pertinencia y coherencia con lo contextual lo cual permitirá lograr la transformación universitaria y el egreso de profesionales con las competencias, conocimientos, habilidades y destrezas para lograr el desarrollo sostenible y sustentable. Pero siempre teniendo presente en el proceso la integración de docentes, estudiantes y sociedad para el logro de la calidad educativa y la transformación de la universidad.

\section{La universidad y la responsabilidad social}

La responsabilidad social en las universidades debe estar en relación directa con los objetivos de cada nación, con las demandas de la sociedad y en coherencia con el proceso educativo universitario. Por lo tanto, referirse a la responsabilidad social de la universidad, es evaluar y replantear su función como formadora de profesionales que deben dar respuesta oportuna y pertinente con el contexto histórico que se vive.

En esta perspectiva, Sánchez (2008) expone que la universidad demanda definir la visión integral de sus funciones académicas sobre un nuevo paradigma donde la comunicación 
oportuna debe ser el eje transformador que le permita, a través de la creación y difusión del conocimiento lograr la correspondencia entre lo que la sociedad demanda por una parte, y la coherencia interna que debe prevalecer en la universidad en la búsqueda de la pertinencia social que aspira el contexto de las instituciones de educación superior, tratando de hacer compatible el discurso con la acción.

Por consiguiente, el lugar estratégico para instaurar y promover la responsabilidad social de la ciencia es justamente la universidad, porque se constituye en el lugar de convergencia entre la producción del saber científico, es decir, la investigación además de la reproducción de este saber lo cual está representado por la transmisión de los conocimientos y la formación de los profesionales que tienen como objetivo socializar las ciencias, las tecnologías y también la información sobre el quehacer científico, de tal manera que la universidad representa un espacio social abierto al debate público.

Es por ello que los Programas Nacionales de Formación del área de la salud buscan la formación de profesionales con pertinencia social, es decir, no sólo la formación universitaria dentro de un área específica de conocimiento sino que facilite el acceso, la inclusión, la equidad y la oportunidad de estudio a todos los ciudadanos, además de dar respuesta a las necesidades y problemáticas que se suceden en comunidades y especialmente para la promoción de la salud y la prevención de las enfermedades a través de la ejecución de proyectos de investigación y extensión universitaria.

\section{Programas Nacionales de Formación del Área de la Salud}

Los Programas Nacionales de Formación (PNF) se definen, según la gaceta oficial 38.930 (2008) como un conjunto de actividades académicas, conducentes a títulos, grados o certificaciones de estudios de educación superior, los cuales fueron creados por iniciativa del ejecutivo nacional, tomando en consideración los lineamientos del plan de desarrollo económico y social de la nación, para ser administrados en distintos espacios educativos en el territorio nacional. 


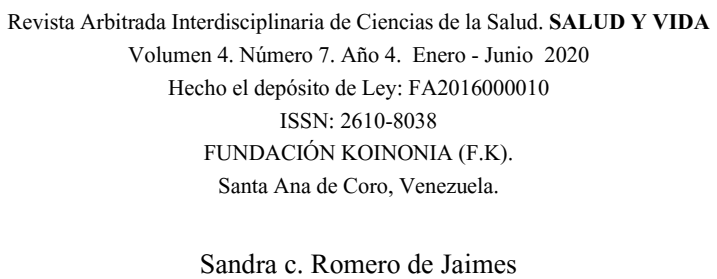

Sandra c. Romero de Jaimes

Cabe señalar, que entre las principales características de los PNF está la formación humanista donde se le da importancia a la formación integral de las personas, considerando, tanto los contenidos programáticos, la experiencia en la adquisición de conocimientos y una perspectiva sustentable. Por consiguiente, los PNF se conciben como el instrumento fundamental para el logro de los objetivos de la Misión Alma Máter, por cuanto las propuestas metodológicas inmersas en su orientación curricular propician la participación, la interacción permanente con las comunidades, la generación de conocimientos basados en la pertinencia social, el intercambio de saberes, la vinculación de la formación y la investigación, objetivos que están en concordancia con el Plan estratégico en la UNEFM.

Es importante señalar, que el enfoque actual de la enseñanza reclama la formación de profesionales capacitados en la elaboración y construcción del conocimiento y la investigación, donde se concibe al conocimiento como un proceso de construcción, convirtiendo al estudiante en el principal responsable de su aprendizaje. Sin embargo, se debe tener muy en cuenta el modelo curricular implementado por lo que se deben realizar revisiones periódicas que permitan discutir y analizar para comprender el accionar pedagógico y constatar si la misión y la visión de los PNF responden con las demandas de la sociedad.

\section{Plan estratégico de la Universidad Nacional Experimental Francisco de Miranda}

La Universidad Nacional Experimental "Francisco de Miranda" (UNEFM), estableció un plan estratégico (PEUNEFM, 2016-2020) para estar en sintonía con los cambios que se promueven en el ámbito universitario, tanto a nivel mundial como a nivel nacional, y poder dar respuesta a las inquietudes y demandas que se requieren para la formación de profesionales integrales y orientados a la potencialización de las capacidades. Se destaca, que este plan tiene su base legal en la Ley Orgánica de Planificación y en la Ley de los Consejos Estadales, y tiene como propósito lograr una articulación efectiva con los lineamientos generales del plan económico y social de la Nación para lograr un ambiente 


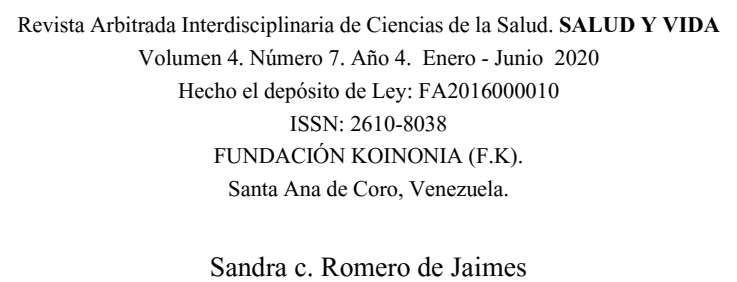

y seguridad en lo que a la educación universitaria se refiere. Se subraya, que el Plan estratégico de la UNEFM, establece como misión:

Ser en el 2020, la universidad de referencia nacional e internacional en la formación de ciudadanos integrales, vinculados con el desarrollo de las potencialidades del territorio en sus distintas escalas; potenciadora de la organización social, económica y cultural tanto en la difusión como en la creación del conocimiento (PEUNEFM2016-2020, p.5).

En esta perspectiva, se establecen como lineamientos curriculares realizar una revisión de los diferentes programas académicos que se imparten en la UNEFM que conlleve a la formación de ciudadanos capaces de redireccionar estrategias que materialicen las políticas de Estado, esto a partir de una transformación moral, ética, epistemológica y pedagógica de todos sus integrantes.

Por otra parte, el Plan Estratégico de la UNEFM busca una formación que sea académica y socialmente pertinente, integradora, de excelencia y calidad, orientada al desarrollo de un sistema de saberes universitario revolucionario, que conjugue el pensamiento científico y los saberes del pueblo.

Por tal motivo, la UNEFM se traza una serie de estrategias:

1.1.4.- Desarrollar un modelo curricular para los programas académicos basado en la complejidad de los conocimientos y saberes objetos de aprendizaje aplicable, mediante el enfoque de competencias.

1.1.5.- Desarrollar y fortalecer técnicas y dinámicas pedagógicas que fomenten el pensamiento crítico y liberador.

1.1.6.- Diseñar e implementar planes para el desarrollo de las competencias de los docentes en nuevos métodos, tecnologías y herramientas de aprendizajes por competencias (PEUNEFM 2016-2020, p.11).

De este modo, la UNEFM busca replantear los paradigmas educativos y orientar la formación del estudiante hacia los conocimientos, habilidades, destrezas y competencias 


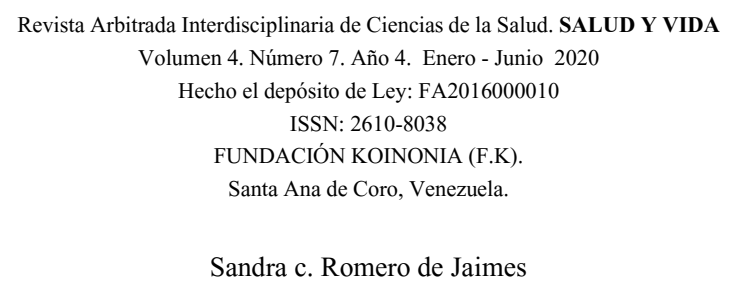

que le permitan desenvolverse de forma eficiente y eficaz en los diferentes ámbitos de trabajo que involucre su acción profesional. Por tal razón, se justifica la implementación de los Programas Nacionales de Formación del área de la salud los cuales además de estar en consonancia con las políticas de estado, tienen como propósito la formación de profesionales con calidad educativa y pertinencia social, que estén comprometidos en el desarrollo sostenible y sustentable de la nación.

\section{METODOLOGIA}

La presente investigación se enmarcó dentro del enfoque epistemológico Racional Deductivo. El tipo de investigación corresponde a un estudio documental donde se planteó como objetivo analizar la calidad educativa y la responsabilidad social de los Programas Nacionales de Formación del área de la salud de la UNEFM a través de la revisión y la información recolectada de los documentos que sirvieron para el análisis y la sistematización del problema de investigación para luego contrastarla con la realidad. Se utilizó la técnica de recolección de información y la revisión documental, tanto de fuentes impresas como electrónicas, además de materiales de referencia que conllevaron a obtener información pertinente para la justificación teórica de la presente investigación lo cual condujo a un análisis de los datos hallados en los documentos informativos, específicamente relacionada con la calidad educativa y la responsabilidad social de los Programas Nacionales de Formación del área de la salud de la UNEFM.

\section{DISCUSIÓN}

La calidad educativa en la educación universitaria está en relación directa con sus objetivos, como lo es la formación de profesionales con los conocimientos, competencias, habilidades y destrezas que permitan el desarrollo individual y colectivo de los ciudadanos. Además, de estar en concordancia con su responsabilidad social que conlleva a la transformación del mundo a partir de la igualdad, la justicia y la paz para el logro de una sociedad más humanizada y poder así alcanzar el desarrollo sostenible y 


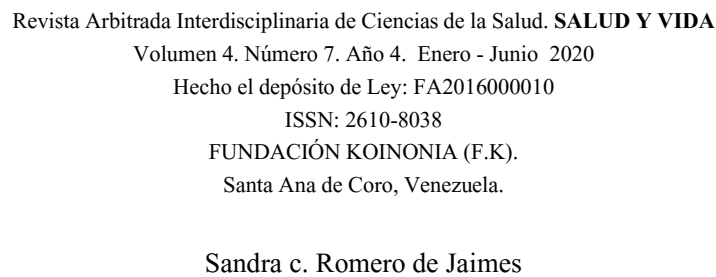

sustentable de las próximas generaciones. De tal manera, que las universidades tienen una responsabilidad que supera el aula de clase y que sólo a través de la implementación de estrategias que revalúen su función y superando paradigmas, se lograrán los cambios que se proyectan en la educación en cuanto a calidad y responsabilidad social para el siglo XXI.

En este sentido, los Programas Nacionales de Formación del área de la salud se constituyen en una política educativa que tiene como propósito dar respuesta a las demandas de la sociedad a través de la formación de profesionales que conlleven al desarrollo sostenible y sustentable de la nación, en concordancia con las nuevas agendas que a nivel internacional se plantean en relación a la educación en el siglo XXI. Sin embargo, se debe tener presente los procesos de reevaluación a nivel académico que conlleven a reformular sus propósitos y poder cumplir con la formación con calidad educativa y pertinencia social del futuro egresado profesional.

Se evidenció durante el análisis que existen debilidades en los PNF del área de la salud que afectan de forma directa la calidad educativa y la pertinencia social del conocimiento pero con revisiones periódicas y disposición se pueden superar para lograr los objetivos que plantea la UNEFM en su plan estratégico y que están en correspondencia con el plan de la nación y las exigencias que demanda la sociedad especialmente en el área de la salud.

\section{CONCLUSIONES}

La revisión documental efectuada constató que no se realizan de forma periódica evaluaciones curriculares de los Programas Nacionales de Formación del área de la salud factor que afecta directamente la calidad educativa.

Se identificaron que los procesos de evaluación de los Programas Nacionales de Formación del área de la salud de la UNEFM en cuanto a su pertinencia social están sustentados por estudios de factibilidad, donde incorporan las necesidades de las 
comunidades como los factores organizacionales y de operatividad académica y administrativa del programa.

Los fundamentos y principios que caracterizan los Programas Nacionales de Formación del área de la salud en cuanto a su pertinencia social, están representados por las potencialidades de las comunidades en cuanto a desarrollo endógeno y a la ausencia de profesionales que contribuyan con el desarrollo sostenible y sustentable.

Existen debilidades en aspectos académicos que influyen de forma negativa en la formación del futuro profesional.

\section{REFERENCIAS CONSULTADAS}

1. Alvarado, P. (2011). La acreditación en los procesos de garantía de calidad en Europa y México. Didac 58. Pág.; 11-17.

2. Ausjal, (2001). Responsabilidad Social de la Universidad.

3. Conferencia Regional de América Latina y el Caribe (2008). Organización de Estados Iberoamericanos (OEI) http://www.oei.es/historico/salactsi/cres.htm. [Consultado; 2017, agosto 21].

4. Asamblea Nacional Constituyente (1999) Constitución de la República Bolivariana de Venezuela. Editorial Las Piedras.

5. Asamblea Nacional Constituyente (2001-2007) Plan de Desarrollo Económico y Social de la Nación.

6. Asamblea Nacional Constituyente (2007-2013) Plan de Desarrollo Económico y Social de la Nación.

7. Ramsden, Paul (2007), Learning to Teach in Higher Education, Londres, Routledge Falmer.

8. Sánchez, A., (2008). Los Programas Nacionales de Formación.

9. UNESCO (1998). La Educación en el Siglo XXI. Visión y Acción, Informe de la Conferencia Mundial sobre Educación Superior. Revista Iberoamericana de Educación [Revista en Línea]

Disponible 
Revista Arbitrada Interdisciplinaria de Ciencias de la Salud. SALUD Y VIDA

Volumen 4. Número 7. Año 4. Enero - Junio 2020

Hecho el depósito de Ley: FA2016000010

ISSN: 2610-8038

FUNDACIÓN KOINONIA (F.K)

Santa Ana de Coro, Venezuela.

Sandra c. Romero de Jaimes

http://www.unesco.cl/pdf/actyeven/ppe/boletin/artesp/47-6.pdf.[Consultado:2017, julio 21].

10. UNESCO (2015). Agenda 2030 para el Desarrollo Sostenible. Disponible en: http://unesdoc.unesco.org/images/0023/002332/233245s.pdf. [Consultado; 2017, agosto 22].

11. Universidad Nacional Experimental "Francisco de Miranda" (2016-2020) Plan Estratégico.

(C2020 por los autores. Este artículo es de acceso abierto y distribuido según los términos y condiciones de la licencia Creative Commons Atribución-NoComercial-Compartirlgual 4.0 Internacional (CC BY-NC-SA 4.0)

(https://creativecommons.org/licenses/by-nc-sa/4.0/). 